Online

ISSN 1600-5368

\title{
Conformational isomerism in 3-(Z)-isobutylidene-1-acetylpiperazine-2,5-dione
}

\section{Wendy A. Loughlin, Michelle A. McCleary and Peter C. Healy}

Copyright (C) International Union of Crystallography

Author(s) of this paper may load this reprint on their own web site provided that this cover page is retained. Republication of this article or its storage in electronic databases or the like is not permitted without prior permission in writing from the IUCr. 
Acta Crystallographica Section E

Structure Reports

Online

ISSN 1600-5368
Wendy A. Loughlin, Michelle A. McCleary and Peter C. Healy*

School of Science, Griffith University, Nathan, Brisbane 4111, Australia

Correspondence e-mail: p.healy@sct.gu.edu.au

\section{Key indicators}

Single-crystal X-ray study

$T=295 \mathrm{~K}$

Mean $\sigma(\mathrm{C}-\mathrm{C})=0.004 \AA$

$R$ factor $=0.053$

$w R$ factor $=0.188$

Data-to-parameter ratio $=14.2$

For details of how these key indicators were automatically derived from the article, see http://journals.iucr.org/e.

\section{Conformational isomerism in 3-(Z)-isobutyl- idene-1-acetylpiperazine-2,5-dione}

The title compound, $\mathrm{C}_{10} \mathrm{H}_{14} \mathrm{~N}_{2} \mathrm{O}_{3}$, (I), is a key intermediate in the synthesis of the cyclic dehydropeptide albonoursin, which has been found to exhibit useful biological and pharmacological properties. It crystallizes with two molecules in the asymmetric unit with different conformational structures. The methylpropylidene side chain is found to be in the $Z$ configuration about the $\mathrm{C}=\mathrm{C}$ double bond for both molecules.

\section{Comment}

Several cyclic dehydropeptides, such as roquefortine (Barrow et al., 1979; Chen \& Joullie, 1998) and albonoursin (Shin et al., 1980) exhibit biological and pharmacological properties. In particular, interest in the latter has led to the syntheses and assignment of configurations of albonoursin and its geometric isomers (Villemin \& Ben Alloum, 1990; Shin et al., 1977). The title monoalkylidene piperazine-2,5-dione, (I), is a key intermediate in the synthesis of albonoursin. The solution structure determination of (I) has relied on the assignment of the configuration of the double bond by NMR spectroscopy (Shin et al., 1977; Gallina \& Liberatori, 1974). We report here the first solid-state structural elucidation of (I), which allows direct correlation between the solution and solid-state structures for assignment of the double-bond geometry. In addition, this represents one of the first accounts of a solid-state structure for a monoalkylidene piperazine-2,5-dione. Compound (I) was obtained by a modification of a procedure (Gallina \& Liberatori, 1973) where 1,3-diacetylpiperazine-2,5dione (Marcuccio \& Elix, 1984) was condensed with 2-methylpropionaldehyde in the presence of a strong base, namely potassium tert-butoxide.
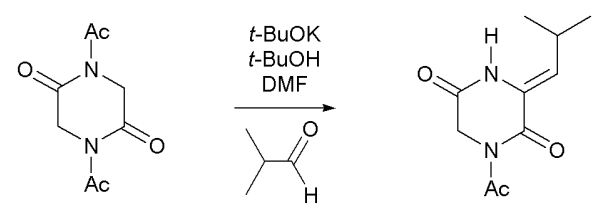

(I)
C 2003 International Union of Crystallography Printed in Great Britain - all rights reserved
Received 8 October 2003 Accepted 17 October 2003 Online 23 October 2003
The crystal structure of (I) contains two independent molecules ( $\mathrm{I} a$ and $\mathrm{I} b)$ in the asymmetric unit, the structures of which are shown in Fig. 1. In both molecules, the methylpropylidene side chain adopts a $Z$ configuration about the double bond, confirming the NMR assignment in the solution state. Both molecules associate as pairs of centrosymmetrically related molecules through classical $R_{2}^{2}(8)$ (Bernstein et al., 1995) $\mathrm{N}-\mathrm{H} \cdots \mathrm{O}$ hydrogen-bonding interactions between $\mathrm{N} 2$ and $\mathrm{O} 3$ [for molecule $\mathrm{I} a$ : $\mathrm{H} \cdots \mathrm{O} 3 A^{\mathrm{i}}=2.00 \AA$, $\mathrm{N} 2 A \cdots \mathrm{O} 3 A^{\mathrm{i}}=$ 


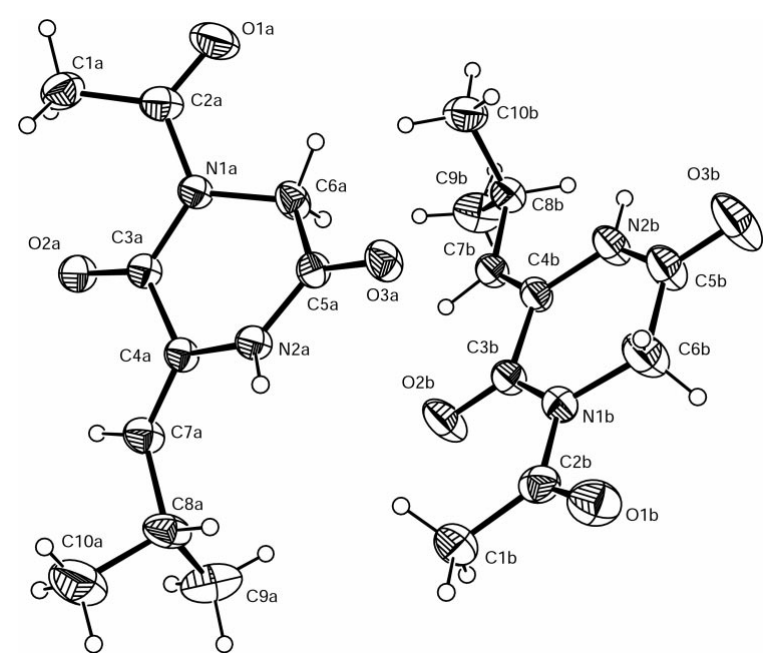

Figure 1

ORTEP-3 (Farrugia, 1997) plot, showing the atomic numbering scheme for molecules ( $\mathrm{I} a$ ) and $(\mathrm{I} b)$. Displacement ellipsoids for non-H atoms are drawn at the $30 \%$ probability level.
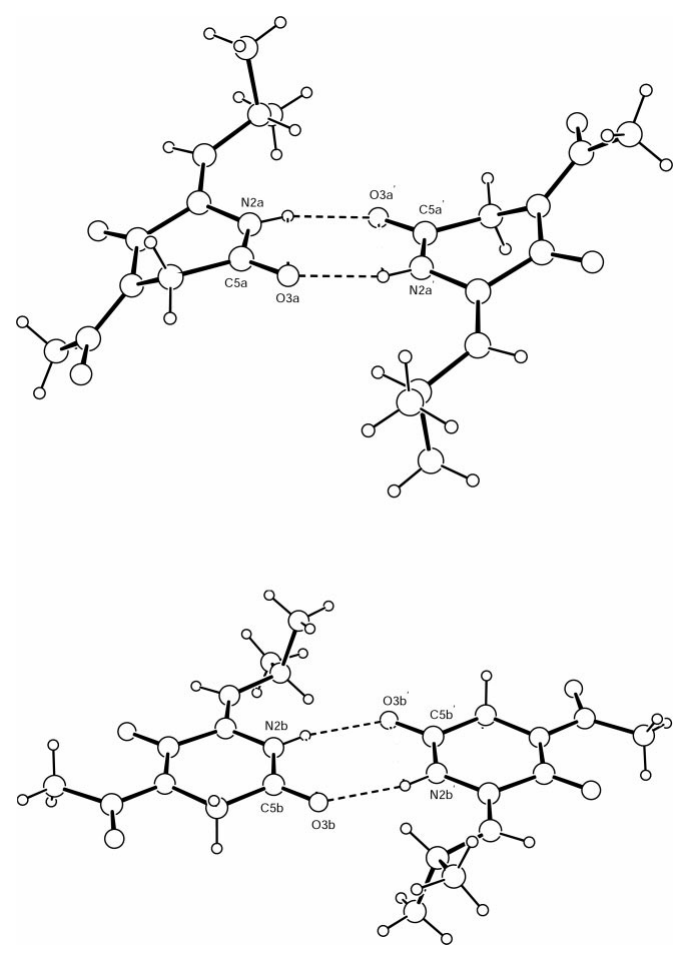

Figure 2

ORTEP-3 plot showing the hydrogen-bonding arrangement for molecules ( $1 a)$ (above) and (I $b)$ (below).

$2.836(2) \AA$ and $\mathrm{N} 2 A-\mathrm{H} \cdots \mathrm{O} 3 A^{\mathrm{i}}=167^{\circ}$; symmetry code: (i) $-x, 2-y, 2-z$; for molecule $\mathrm{I} b: \mathrm{H} \cdots \mathrm{O} 3 B^{\mathrm{ii}}=2.07 \AA$, $\mathrm{N} 2 B \cdots \mathrm{O} 3 B^{\mathrm{ii}}=2.908(3) \AA$ and $\mathrm{N} 2 B-\mathrm{H} \cdots \mathrm{O} 3 B^{\mathrm{ii}}=167^{\circ}$; symmetry code: (ii) $-x, 1-y, 1-z]$ (Fig. 2). This hydrogenbonding feature is observed in the structures of other piperazinedione systems (Ongania et al., 1985; Ajo et al., 1985).

The piperazinedione ring in molecule ( $\mathrm{I} a$ ) adopts a puckered conformation with the peripheral isopropyl and keto substituents lying well out of the plane defined by the inter- molecular $\mathrm{N}-\mathrm{H} \cdots \mathrm{O}$ hydrogen bonding. In the crystal lattice (Fig. 3), the dimers of ( $\mathrm{I} a$ ) lie approximately in the $a c$ plane at $b \sim 0$, with the long axis of the dimer oriented along the crystallographic $a$ axis. In contrast, the conformational structure of $(\mathrm{I} b)$ is essentially planar for all non-H atoms other than the $\mathrm{C} 9$ and $\mathrm{C} 10$ methyl groups. As in (I $a)$, the dimers of (I $b$ ) lie approximately in the $a c$ plane but at $b \sim \frac{1}{2}$, with the long axis oriented along the $c$ axis. The bond lengths for both molecules (Table 1) are in accord with conventional values (Allen et al., 1987), with differences between corresponding values in the two molecules no greater than $0.01 \AA$. Greater diversity is apparent in the bond angles, with the greatest differences observed for the angles about N1 and C6.

\section{Experimental}

Potassium tert-butoxide $(0.567 \mathrm{~g}, 5.053 \mathrm{mmol})$ in tert-butanol $(10 \mathrm{ml}$, $0.105 \mathrm{~mol})$ was added to a stirred solution of 2-methylpropionaldehyde $(1.80 \mathrm{ml}, 0.020 \mathrm{~mol})$ and 1,4-diacetylpiperazine-2,5-dione $(1.054 \mathrm{~g}, 5.328 \mathrm{mmol})$ in dry DMF $(10.50 \mathrm{ml}, 0.136 \mathrm{~mol})$ at $273 \mathrm{~K}$. The mixture was stirred at room temperature for $24 \mathrm{~h}$ and worked up as described elsewhere (Gallina \& Liberatori, 1973). The crude product was recrystallized (ethyl acetate/hexane), giving (I) as white crystals (263 mg, 24\%). Colourless crystals of (I) [m.p. 419.9-420.3 K; literature 419.2-420.2 K (Gallina \& Liberatori, 1973, 1974) and 423.2424.2 K (Shin et al., 1977)] were isolated by slow evaporation of a hexane-ethyl acetate solution of (I). Analysis found: C 57.41, H 6.77, $\mathrm{N} 13.45 \%$; calculated for $\mathrm{C}_{10} \mathrm{H}_{14} \mathrm{~N}_{2} \mathrm{O}_{3}$ : C 57.13, $\mathrm{H} 6.71, \mathrm{~N} 13.33 \%$. $v_{\max }(\mathrm{KBr}) / \mathrm{cm}^{-1}: 3390,3196(\mathrm{NH}), 1687,1650,(\mathrm{CO}), 1636,(\mathrm{C}=\mathrm{C})$. $\delta_{H}\left(200 \mathrm{MHz}, \mathrm{CDCl}_{3}\right.$, p.p.m.): $8.10(1 \mathrm{H}$, brs, $\mathrm{NH}), 6.18\left(1 \mathrm{H}, d, J_{7,8}=10\right.$ $\mathrm{Hz}, 7-\mathrm{H}), 4.44(2 \mathrm{H}, s, 6-\mathrm{H}), 2.75-2.60(1 \mathrm{H}, m, 8-\mathrm{H}), 2.61(3 \mathrm{H}, s, 1-\mathrm{H})$, $1.10\left(6 \mathrm{H}, d, J_{9 / 10,8}=6.5 \mathrm{~Hz}, 9-\mathrm{H}, 10-\mathrm{H}\right) . \delta_{C}\left(50 \mathrm{MHz}, \mathrm{CDCl}_{3}\right.$, p.p.m.): 172.7 (C2), 164.2 (C5), 160.2 (C3), 131.6 (C4), 124.5 (C7), 46.0 (C6), 27.2 (C9 and C10), 25.9 (C8), $22.0(\mathrm{C} 1) . \mathrm{ESMS}^{+}: 209\left(\mathrm{MH}^{+}, 100 \%\right)$, $233\left(\mathrm{MNa}^{+}, 100 \%\right)$.

\section{Crystal data}

$\mathrm{C}_{10} \mathrm{H}_{14} \mathrm{~N}_{2} \mathrm{O}_{3}$

$M_{r}=210.23$

Triclinic, $P \overline{1}$

$a=10.7310(18) \AA$

$b=10.827(3) \AA$

$c=9.7916(14) \AA$

$\alpha=95.607(17)^{\circ}$

$\beta=94.680(13)^{\circ}$

$\gamma=76.484(17)^{\circ}$

$V=1098.8(4) \AA^{3}$

$Z=4$

$D_{x}=1.271 \mathrm{Mg} \mathrm{m}^{-3}$

Mo $\mathrm{K} \alpha$ radiation

Cell parameters from 25

reflections

$\theta=19.4-20.0^{\circ}$

$\mu=0.10 \mathrm{~mm}^{-1}$

$T=295 \mathrm{~K}$

Prism, colourless

$0.60 \times 0.50 \times 0.30 \mathrm{~mm}$

Data collection

Rigaku AFC-7 $R$ diffractometer $\omega-2 \theta$ scans

Absorption correction: none 4100 measured reflections 3872 independent reflections 2691 reflections with $I>2 \sigma(I)$ $R_{\text {int }}=0.019$

$\theta_{\text {max }}=25.0^{\circ}$

$h=0 \rightarrow 12$

$k=-12 \rightarrow 12$

$l=-11 \rightarrow 11$

3 standard reflections every 150 reflections intensity decay: $1.3 \%$

\section{Refinement}

Refinement on $F^{2}$

$R\left[F^{2}>2 \sigma\left(F^{2}\right)\right]=0.053$

$w R\left(F^{2}\right)=0.188$

$S=1.09$

3872 reflections

272 parameters

$\mathrm{H}$-atom parameters constrained

$$
\begin{aligned}
& w=1 /\left[\sigma^{2}\left(F_{o}^{2}\right)+(0.1241 P)^{2}\right. \\
& +0.0666 P] \\
& \text { where } P=\left(F_{o}{ }^{2}+2 F_{c}{ }^{2}\right) / 3 \\
& (\Delta / \sigma)_{\max }<0.001 \\
& \Delta \rho_{\max }=0.26 \mathrm{e}^{-3} \\
& \Delta \rho_{\min }=-0.26 \mathrm{e}^{-3} \\
& \text { Extinction correction: SHELXL97 } \\
& \text { Extinction coefficient: } 0.032 \text { (7) }
\end{aligned}
$$


Table 1

Selected geometric parameters $\left(\AA{ }^{\circ}\right)$.

\begin{tabular}{|c|c|c|c|}
\hline $\mathrm{O} 1 A-\mathrm{C} 2 A$ & $1.210(3)$ & $\mathrm{O} 1 B-\mathrm{C} 2 B$ & $1.214(4)$ \\
\hline $\mathrm{O} 2 A-\mathrm{C} 3 A$ & $1.203(3)$ & $\mathrm{O} 2 B-\mathrm{C} 3 B$ & $1.214(3)$ \\
\hline $\mathrm{O} 3 A-\mathrm{C} 5 A$ & $1.233(3)$ & $\mathrm{O} 3 B-\mathrm{C} 5 B$ & $1.224(4)$ \\
\hline $\mathrm{N} 1 A-\mathrm{C} 2 A$ & $1.408(3)$ & $\mathrm{N} 1 B-\mathrm{C} 6 B$ & $1.458(4)$ \\
\hline $\mathrm{N} 1 A-\mathrm{C} 3 A$ & $1.394(3)$ & $\mathrm{N} 1 B-\mathrm{C} 2 B$ & $1.417(3)$ \\
\hline $\mathrm{N} 1 A-\mathrm{C} 6 A$ & $1.463(3)$ & $\mathrm{N} 1 B-\mathrm{C} 3 B$ & $1.380(3)$ \\
\hline $\mathrm{N} 2 A-\mathrm{C} 4 A$ & $1.408(3)$ & $\mathrm{N} 2 B-\mathrm{C} 5 B$ & $1.328(4)$ \\
\hline $\mathrm{N} 2 A-\mathrm{C} 5 A$ & $1.335(3)$ & $\mathrm{N} 2 B-\mathrm{C} 4 B$ & $1.406(3)$ \\
\hline $\mathrm{C} 1 A-\mathrm{C} 2 A$ & $1.484(4)$ & $\mathrm{C} 1 B-\mathrm{C} 2 B$ & $1.483(4)$ \\
\hline $\mathrm{C} 3 A-\mathrm{C} 4 A$ & $1.501(3)$ & $\mathrm{C} 3 B-\mathrm{C} 4 B$ & $1.495(3)$ \\
\hline $\mathrm{C} 4 A-\mathrm{C} 7 A$ & $1.332(3)$ & $\mathrm{C} 4 B-\mathrm{C} 7 B$ & $1.325(3)$ \\
\hline $\mathrm{C} 5 A-\mathrm{C} 6 A$ & $1.494(3)$ & $\mathrm{C} 5 B-\mathrm{C} 6 B$ & $1.485(4)$ \\
\hline $\mathrm{C} 7 A-\mathrm{C} 8 A$ & $1.500(3)$ & $\mathrm{C} 7 B-\mathrm{C} 8 B$ & $1.505(3)$ \\
\hline $\mathrm{C} 8 A-\mathrm{C} 10 A$ & $1.513(4)$ & $\mathrm{C} 8 B-\mathrm{C} 9 B$ & $1.507(4)$ \\
\hline $\mathrm{C} 8 A-\mathrm{C} 9 A$ & $1.519(5)$ & $\mathrm{C} 8 B-\mathrm{C} 10 B$ & $1.525(4)$ \\
\hline $\mathrm{C} 2 A-\mathrm{N} 1 A-\mathrm{C} 3 A$ & $124.14(18)$ & $\mathrm{C} 2 B-\mathrm{N} 1 B-\mathrm{C} 3 B$ & $125.08(19)$ \\
\hline $\mathrm{C} 2 A-\mathrm{N} 1 A-\mathrm{C} 6 A$ & $117.28(17)$ & $\mathrm{C} 2 B-\mathrm{N} 1 B-\mathrm{C} 6 B$ & $112.6(2)$ \\
\hline $\mathrm{C} 3 A-\mathrm{N} 1 A-\mathrm{C} 6 A$ & $118.47(16)$ & $\mathrm{C} 3 B-\mathrm{N} 1 B-\mathrm{C} 6 B$ & $122.3(2)$ \\
\hline $\mathrm{C} 4 A-\mathrm{N} 2 A-\mathrm{C} 5 A$ & $123.42(17)$ & $\mathrm{C} 4 B-\mathrm{N} 2 B-\mathrm{C} 5 B$ & $125.4(2)$ \\
\hline $\mathrm{O} 1 A-\mathrm{C} 2 A-\mathrm{N} 1 A$ & $118.1(3)$ & $\mathrm{O} 1 B-\mathrm{C} 2 B-\mathrm{N} 1 B$ & $117.6(2)$ \\
\hline $\mathrm{O} 1 A-\mathrm{C} 2 A-\mathrm{C} 1 A$ & $122.7(3)$ & $\mathrm{O} 1 B-\mathrm{C} 2 B-\mathrm{C} 1 B$ & $121.3(2)$ \\
\hline $\mathrm{N} 1 A-\mathrm{C} 2 A-\mathrm{C} 1 A$ & $119.22(19)$ & $\mathrm{N} 1 B-\mathrm{C} 2 B-\mathrm{C} 1 B$ & $121.1(3)$ \\
\hline $\mathrm{N} 1 A-\mathrm{C} 3 A-\mathrm{C} 4 A$ & 114.57 (17) & $\mathrm{N} 1 B-\mathrm{C} 3 B-\mathrm{C} 4 B$ & $117.91(18)$ \\
\hline $\mathrm{O} 2 A-\mathrm{C} 3 A-\mathrm{N} 1 A$ & 123.49 (19) & $\mathrm{O} 2 B-\mathrm{C} 3 B-\mathrm{N} 1 B$ & $122.0(2)$ \\
\hline $\mathrm{O} 2 A-\mathrm{C} 3 A-\mathrm{C} 4 A$ & $121.94(18)$ & $\mathrm{O} 2 B-\mathrm{C} 3 B-\mathrm{C} 4 B$ & $120.1(2)$ \\
\hline $\mathrm{N} 2 A-\mathrm{C} 4 A-\mathrm{C} 3 A$ & 117.33 (17) & $\mathrm{N} 2 B-\mathrm{C} 4 B-\mathrm{C} 3 B$ & $117.90(19)$ \\
\hline $\mathrm{N} 2 A-\mathrm{C} 4 A-\mathrm{C} 7 A$ & 123.57 (19) & $\mathrm{N} 2 B-\mathrm{C} 4 B-\mathrm{C} 7 B$ & $122.30(19)$ \\
\hline $\mathrm{C} 3 A-\mathrm{C} 4 A-\mathrm{C} 7 A$ & $118.96(19)$ & $\mathrm{C} 3 B-\mathrm{C} 4 B-\mathrm{C} 7 B$ & $119.80(19)$ \\
\hline $\mathrm{O} 3 A-\mathrm{C} 5 A-\mathrm{N} 2 A$ & $123.26(19)$ & $\mathrm{O} 3 B-\mathrm{C} 5 B-\mathrm{N} 2 B$ & $123.4(3)$ \\
\hline $\mathrm{N} 2 A-\mathrm{C} 5 A-\mathrm{C} 6 A$ & $115.91(18)$ & $\mathrm{N} 2 B-\mathrm{C} 5 B-\mathrm{C} 6 B$ & $118.8(3)$ \\
\hline $\mathrm{O} 3 A-\mathrm{C} 5 A-\mathrm{C} 6 A$ & $120.83(18)$ & $\mathrm{O} 3 B-\mathrm{C} 5 B-\mathrm{C} 6 B$ & $117.8(3)$ \\
\hline $\mathrm{N} 1 A-\mathrm{C} 6 A-\mathrm{C} 5 A$ & $113.11(17)$ & $\mathrm{N} 1 B-\mathrm{C} 6 B-\mathrm{C} 5 B$ & $117.3(3)$ \\
\hline $\mathrm{C} 4 A-\mathrm{C} 7 A-\mathrm{C} 8 A$ & $128.6(2)$ & $\mathrm{C} 4 B-\mathrm{C} 7 B-\mathrm{C} 8 B$ & $127.71(19)$ \\
\hline $\mathrm{C} 7 A-\mathrm{C} 8 A-\mathrm{C} 9 A$ & $109.8(2)$ & $\mathrm{C} 7 B-\mathrm{C} 8 B-\mathrm{C} 9 B$ & $111.14(19)$ \\
\hline $\mathrm{C} 7 A-\mathrm{C} 8 A-\mathrm{C} 10 A$ & $110.1(3)$ & $\mathrm{C} 7 B-\mathrm{C} 8 B-\mathrm{C} 10 B$ & $110.18(19)$ \\
\hline $\mathrm{C} 9 A-\mathrm{C} 8 A-\mathrm{C} 10 A$ & $109.3(2)$ & $\mathrm{C} 9 B-\mathrm{C} 8 B-\mathrm{C} 10 B$ & $110.4(2)$ \\
\hline
\end{tabular}

$\mathrm{H}$ atoms were constrained in the riding-model approximation, with $\mathrm{C}-\mathrm{H}$ distances set at $0.95 \AA$ and $\mathrm{N}-\mathrm{H}$ set at $0.85 \AA$. $U_{\text {iso values for }}$ the $\mathrm{H}$ atoms were set at $1.2 U_{\text {eq }}$ of the parent atom.

Data collection: MSC/AFC-7 Diffractometer Control Software (Molecular Structure Corporation, 1999); cell refinement: $M S C / A F C$ 7 Diffractometer Control Software; data reduction: TEXSAN for Windows (Molecular Structure Corporation, 1997-2001); program(s) used to solve structure: TEXSAN for Windows; program(s) used to refine structure: TEXSAN for Windows and SHELXL97 (Sheldrick, 1997); molecular graphics: PLATON (Spek, 2001) and ORTEP-3 (Farrugia, 1997); software used to prepare material for publication: TEXSAN for Windows and PLATON.

The authors thank Griffith University for financial assistance.

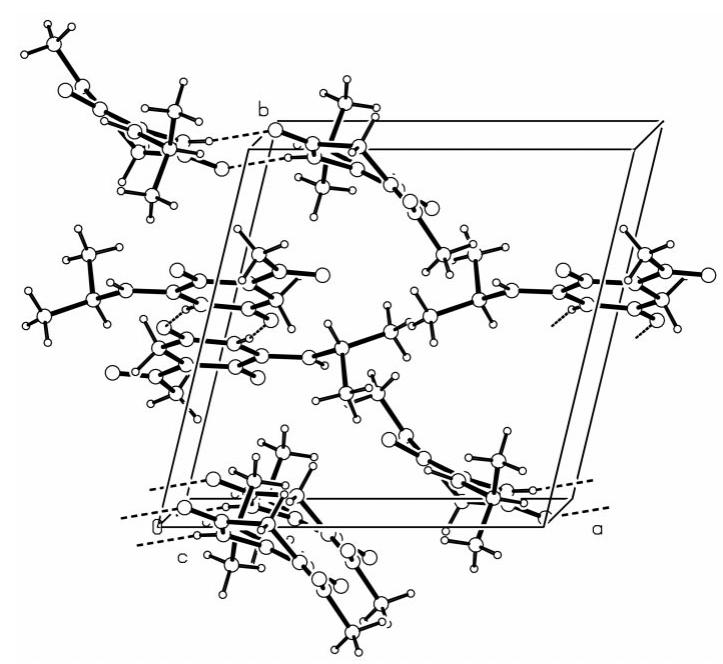

Figure 3

Crystal packing diagram for (I), viewed down the $c$ axis with $a$ horizontal and $b$ vertical.

\section{References}

Ajo, D., Casarin, M., Bertoncello, R., Busetti, V., Ottenheijm, H. C. J. \& Plate, R. (1985). Tetrahedron, 41, 5543-5552.

Allen, F. H., Kennard, O., Watson, D. G., Brammer, L., Orpen, A. G. \& Taylor, R. (1987). J. Chem. Soc. Perkin Trans. 2, pp. S1-19.

Barrow, K. D., Colley, P. W. \& Tribe, D. E. (1979). Chem. Commun. pp. 225226.

Bernstein, J., Davis, R. E., Shimoni, L. \& Chang, N.-L. (1995). Angew. Chem Int. Ed. Engl. 34, 1555-1573.

Chen, W. C. \& Joullie, M. M. (1998). Tetrahedron Lett. 39, 8401-8404.

Farrugia, L. J. (1997). J. Appl. Cryst. 30, 565.

Gallina, C. \& Liberatori, A. (1973). Tetrahedron Lett. 14, 1135-1136.

Gallina, C. \& Liberatori, A. (1974). Tetrahedron, 30, 667-673.

Marcuccio, S. M. \& Elix, J. A. (1984). Aust. J. Chem. 37, 1791-1794.

Molecular Structure Corporation (1999). MSC/AFC-7 Diffractometer Control for Windows. Version 1.02. MSC, 9009 New Trails Drive, The Woodlands, TX 77381, USA.

Molecular Structure Corporation (1997-2001). TEXSAN for Windows. Version 1.06. MSC, 9009 New Trails Drive, The Woodlands, TX 77381, USA

Ongania, K. H., Granozzi, G., Busetti, V., Casarin, M. \& Ajo, D. (1985). Tetrahedron, 41, 2015-2018.

Sheldrick, G. M. (1997). SHELXL97. University of Göttingen, Germany.

Shin, C., Hayakawa, M., Kato, H., Mikami, K. \& Yoshimura, J. (1980). J. Chem. Soc. Perkin Trans. 1. pp. 419-421, and references therein.

Shin, C.-G., Hayakawa, M., Mikami, K. \& Yoshimura, J. (1977). Tetrahedron Lett. 18, 863-866.

Spek, A. L. (2001). PLATON for Windows. Version 121201 University of Utrecht, The Netherlands.

Villemin, D. \& Ben Alloum, A. (1990). Synth. Commun. pp. 3325-3321. 